\title{
Hemangioblastoma sólido-quístico supratentorial afectando la hoz del cerebro. Presentación de un caso
}

\author{
A. Elguezabal; M.L. Díaz; J. Landeyro; M. Gené; L. Boutayeb; M. Escosa* y J.J. Sirvent
}

Servicios de Anatomía Patológica y de Neurocirugía*. Hospital Universitari Joan XXIII, Tarragona.

Resumen

Introducción. Los hemangioblastomas son neoplasias benignas que se originan en el sistema nervioso central y constituyen entre un $\mathbf{1 , 5 - 2 , 5 \%}$ de los tumores intracraneales. Mayoritariamente son de localización infratentorial, afectando principalmente al cerebelo (76\%). Las lesiones supratentoriales son sumamente raras, siendo en estos casos la localización más habitual el lóbulo frontal, parietal o temporal. La afectación meníngea es excepcional, habiéndose descrito sólo ocho casos en la literatura. En un 30\% de los casos, estos tumores se asocian al síndrome de von Hippel Lindau (VHL).

Caso clínico. Mujer de 67 años sin antecedentes patológicos ni familiares de interés que se presentó con clínica neurológica de $\mathbf{4}$ meses de evolución. El estudio de resonancia magnética craneal demostró una lesión única sólido-quística frontal paramedial derecha, en contacto con la hoz del cerebro, que se orientó como meningioma. El estudio anatomo-patológico de la pieza quirúrgica objetivó una proliferación celular constituida por células poligonales con citoplasma claro debido a la presencia de vacuolas intracitoplasmáticas y núcleo redondo u oval sin atipia citológica. Estas células estaban acompañadas de una rica red vascular de tipo capilar, con anastomosis y extravasación sanguínea. Se diagnosticó de hemangioblastoma supratentorial.

Discusión. El diagnóstico preoperatorio de estas neoplasias es difícil debido a que la sospecha clínica es baja cuando se halla en localización supratentorial. Las técnicas de imagen son de utilidad, realizándose el diagnóstico definitivo a través del estudio anatomopatológico. El uso de técnicas de inmunohistoquímica es de gran ayuda para el diagnóstico diferencial con lesiones que habitualmente se localizan en esta región. La importancia de un diagnóstico correcto de estos tumores histológicamente benignos, radica, entre otras cosas, en la posible asociación con en síndrome de VHL y sus complicaciones.

Recibido: 26-01-10. Aceptado: 12-07-10
PALABRAS CLAVE: Hemangioblastoma supratentorial. Hoz del cerebro. Tumor del Sistema Nervioso Central. Von Hippel Lindau.

Solid-cystic supratentorial hemangioblastoma affecting the falx cerebri. Report of a case

Summary

Introduction. Hemangioblastomas are benign neoplasias that are originated in the central nervous system and constitute between $\mathbf{1 . 5 - 2 . 5 \%}$ of intracranial tumors. The majority of them are infratentorial, mainly affecting the cerebellum (76\%). Supratentorial lessions are rare, being in these cases the frontal, parietal or temporal lobes the most common locations. Meningeal involvement is infrequent. Only eight cases have been reported in the literature. In $30 \%$ of the cases, these tumors are associated with von Hippel Lindau syndrome (VHL).

Case report. 67 year old woman without any medical or family history. She presented with 4 month evolution neurological symptoms. The cranial MRI scan showed a solitary solid-cystic lesion on the right paramedian frontal lobe, in contact with the falx cerebri. The pathological analysis showed a cellular proliferation composed of polygonal cells with clear cytoplasm due to the presence of intracytoplasmic vacuoles and round or oval nucleus without cytologic atypia. These cells were accompanied by a rich vascular network of capillary type and blood extravasation. She was diagnosed of supratentorial hemangioblastoma.

Conclusion. The preoperative diagnosis of these neoplasms is difficult because the clinical suspicion is low in supratentorial location. Imaging techniques are useful but definitive diagnosis is made through pathologic examination. The use of immunohistochemical techniques is helpful for the differential diagnosis with lesions that are more common in this region. The importance of a correct diagnosis of these histologically benign tumors, lies on the possible association with VHL syndrome and its complications. 


\section{Introducción}

Los hemangioblastomas son neoplasias benignas que se originan en el sistema nervioso central y constituyen entre un $1,5-2,5 \%$ de los tumores intracraneales s $^{2,3,4,9}$. Mayoritariamente son infratentoriales, siendo el sitio más frecuentemente afectado el cerebelo (76\%). En un 30\% de los casos, estos tumores se asocian al síndrome de von Hippel Lindau (VHL). Este es un trastorno autosómico dominante debido a la mutación del gen supresor localizado en el cromosoma 3p25-26, que se caracteriza por la asociación de hemangioblastomas del sistema nervioso central (SNC) y retina, carcinoma de células renales, quistes viscerales, feocromocitoma adrenal y cistadenoma papilar del epidídimo ${ }^{2,3,4,6,8,9,10}$. La localización supratentorial es sumamente rara y dificulta el diagnóstico preoperatorio debido a la baja sospecha clínico-radiológica. Asimismo, la afectación meníngea es excepcional, encontrándose pocos casos descritos en la literatura ${ }^{2,3,4,6,9}$.

\section{Presentación del caso}

Mujer de 67 años con antecedentes personales de hipertensión arterial y artrosis y sin antecedentes familiares de importancia, que acudió a urgencias por clínica neurológica de 4 meses de evolución. Los familiares refirieron un empeoramiento progresivo con torpeza al caminar, pérdida de equilibrio y caídas frecuentes, asociado a trastornos del ánimo, decaimiento general y pensamientos pesimistas.

Al ingreso la paciente se encontraba en Glasgow 15, destacaba una desorientación en el tiempo, bradipsiquia y dispraxia para órdenes simples, sin observarse afasia. A la exploración física se objetivó una marcha insegura con pequeños pasos y disminución del braceo, tendencia a desviarse hacia a la izquierda, hemiparesia 4/5 izquierda, bradicinesia e hiperreflexia global de predominio en extremidades inferiores. Se realizó una resonancia magnética (RM) craneal en la que se observó una lesión expansiva con áreas en su interior de aspecto quístico, localizada en la región frontal paramedial derecha en contacto con el tercio anterior de la hoz del cerebro. Presentaba abundante edema perilesional y efecto de masa que desplazaba las estructuras de la línea media produciendo una herniación subfalcial y uncal (figura 1). Se informó como compatible con meningioma y se intervino quirúrgicamente practicándose una craneotomía frontal izquierda con resección total de la lesión. En el laboratorio de anatomía patológica se recibió una muestra constituida por 4 fragmentos de coloración parduzca y aspecto hemático que en su conjunto median $40 \mathrm{~mm}$. Microscópicamente se observó una proliferación celular conformada por células poligonales, citoplasma claro con vacuolas intracitoplasmáticas y núcleo redondo

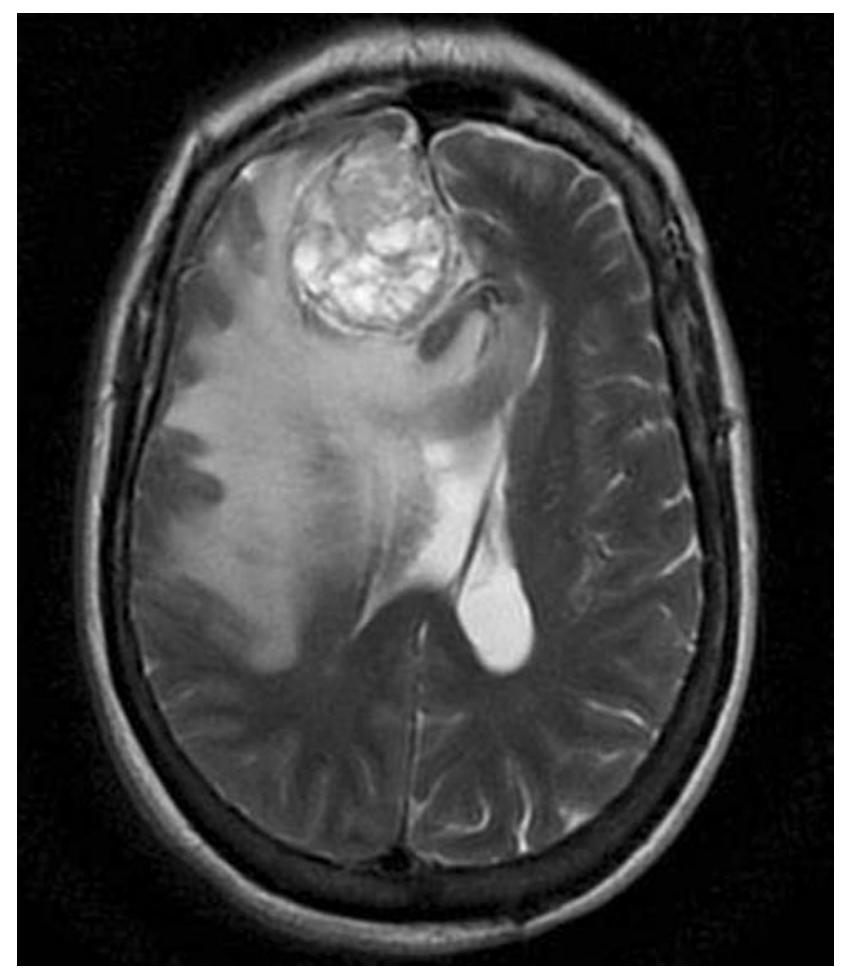

Figura1. Resonancia mágnética. Lesión expansiva sólidoquística en región frontal paramedial derecha.

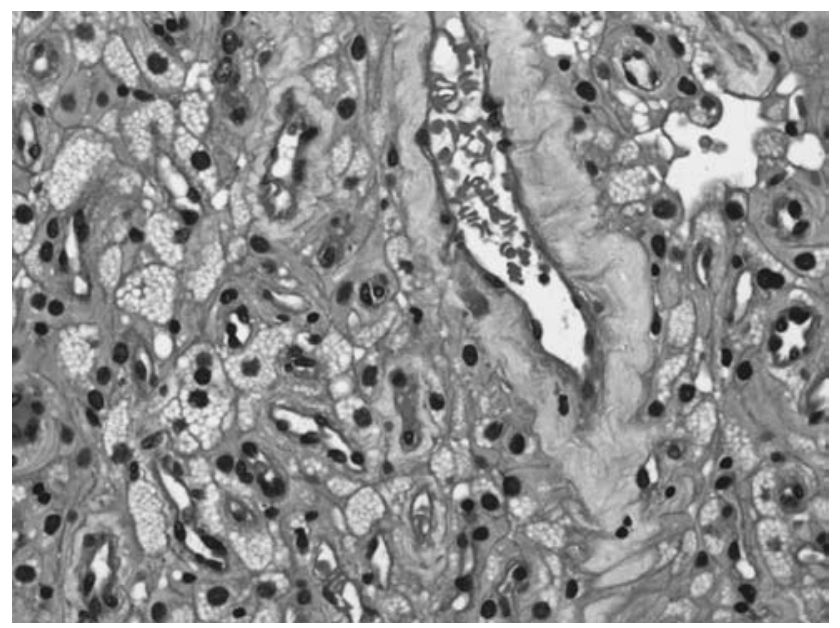

Figura 2. Hematoxilina-Eosina 100x. Red capilar formada por células endoteliales prominentes, entre las que se observan células estromales con citoplasma claro vacuolado.

u oval sin atipia citológica. Estas células estaban acompañadas de una rica red vascular de tipo capilar, con áreas de anastomosis y extravasación sanguínea (figuras 2 y 3). El estudio inmunohistoquímico demostró positividad de las células tumorales para enolasa neuronal específica (NSE) y vimentina, siendo negativa par citoqueratinas de amplio espectro, CD10, proteína glial acídica fibrilar (GFAP), S100, antígeno de membrana epitelial (EMA) 


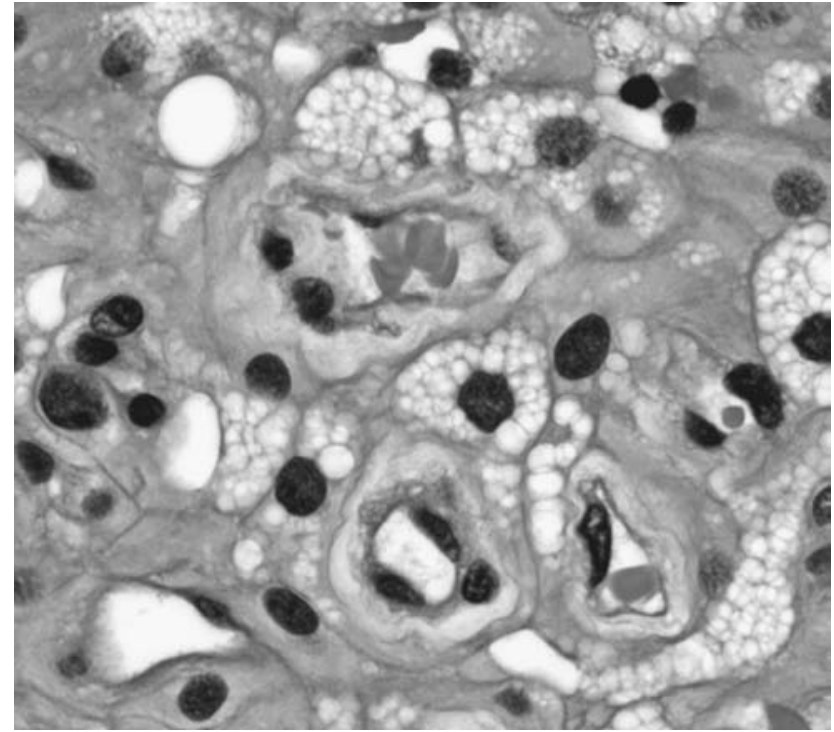

Figura 3. Hematoxilina-Eosina 200x. Detalle de células estromales.

y antígeno carcinoembrionario (CEA). Asimismo las células del endotelio vascular resultaron positivas para CD31 y CD34. Se diagnosticó de hemangioblastoma de localización intracraneal frontal derecha. La evolución postoperatoria fue favorable, manteniéndose en Glasgow 15 sin observarse signos de foco. En los controles posteriores, el examen clínico y estudio por RM descartaron signos de recidiva local. Asimismo se descartaron lesiones en otros órganos sugestivas de síndrome VHL y los estudios realizados a familiares directos excluyeron también dicho síndrome.

\section{Discusión}

Los hemangioblastomas son neoplasias benignas vasculares de origen desconocido que tienen mayor prevalencia en el sexo masculino y suelen ocurrir en la cuarta o quinta década de la vida, sin embargo se pueden presentar a cualquier edad e incluso se han descrito casos de lesiones congénitas ${ }^{1,5,7}$. Estas lesiones se pueden encontrar en diversas localizaciones del sistema nervioso central, constituyendo entre un 1,5-2,5\% de todas las neoplasias del SNC y entre un $7-12 \%$ de las halladas en la fosa posterior. $\mathrm{La}$ localización supratentorial es infrecuente y en estos casos suelen encontrarse por orden de frecuencia en el lóbulo frontal, parietal o temporal ${ }^{2,3,4,9}$. La afectación meníngea es excepcional, habiéndose descrito ocho casos en la literatura. Todos ellos presentaban consistencia sólida y sólo 3 estaban localizados en el lóbulo frontal ${ }^{1,9,10}$. Un $30 \%$ de los casos se asocia al síndrome de VHL, y en estos, el $80 \%$ corresponden a lesiones aisladas. El 70\% restante se presenta de forma espontánea y solo un 5\% de estas neoplasias son múltiples, porcentaje que es aún menor cuando se trata de presentación supratentorial $1^{4,9}$.

Las manifestaciones clínicas suelen ser signos de hipertensión endocraneana, epilepsia o hemiparesias. La hemorragia intracerebral o subaracnoidea son excepcionales $\mathrm{e}^{2,3,4,8}$.

El diagnóstico preoperatorio a través de técnicas de imagen es difícil. Los estudios como la RM muestran lesiones que, a pesar de tener las mismas características que las halladas en fosa posterior, rara vez se sospechan cuando se encuentran en localización supratentorial. Además, la afectación meníngea, como en nuestro caso, puede llevar al error diagnóstico de meningioma ${ }^{2,3,4,6}$. El diagnóstico definitivo se realiza mediante el estudio anatomo-patológico. Macroscópicamente, son lesiones bien delimitadas, aunque no encapsuladas, que pueden invadir el tejido circundante de forma ocasional. Pueden ser sólidos o quísticos o estar formados por ambos componentes simultáneamente. Microscópicamente se caracterizan por estar constituidos por células estromales de citoplasma claro vacuolado, sumergidas en una rica red capilar formada por células endoteliales prominentes que presentan inmunorreactividad para CD31 y CD34. Las células estromales se consideran el componente neoplásico de estos tumores. Muestran núcleos hipercromáticos con atipia discreta ocasional, bajo índice mitótico y carecen de organelas u otro accesorio específico. Presentan inmunorreactividad focal para NSE y variable para proteína $\mathrm{S} 100$, siendo habitualmente negativo para EMA. La positividad para GFAP se ha asociado a cambios reactivos de los astrocitos, más que con las células estromales propiamente dichas ${ }^{2,3,6,9,10}$. Se describen 2 variantes histológicas: la reticular en donde las células tumorales se distribuyen alrededor de la red vascular, y la celular, donde dichas células forman grandes nidos o sábanas ${ }^{3,5,6}$.

En el diagnóstico diferencial a través del estudio histológico, se deben tener en cuenta en primer lugar las metástasis de tumores de células claras, principalmente cuando las lesiones son múltiples. En este caso el más importante es el carcinoma de células renales. Este se diferencia por la atipia citológica y por la positividad para vimentina, $\mathrm{CD} 10$ y citoqueratinas. Además hay que tener en cuenta tumores primarios del SNC con componente vascular importante, o con presencia de células claras. En el primer caso cabe mencionar al meningioma angiomatoso y al hemangiopericitoma. El meningioma angiomatoso se caracteriza por tener vasos sanguíneos pequeños y dilatados en un medio constituido por células meningoteliales típicas positivas para EMA y vimentina. El hemangiopericitoma está compuesto por vasos sanguíneos con endotelio aplanado y por células con abundante citoplasma, núcleo oval y pequeño con discreto pleomorfismo que son positivas para bcl2, CD99, factor XIIIa, vimentina y EMA. Dentro 
de los tumores primarios del SNC con células claras, se debe excluir el meningioma de células claras, el cual está constituido por células de citoplasma vacuolado PAS positivo y comparten el inmunofenotipo de los meningiomas convencionales.

Una vez realizado el diagnóstico definitivo de hemangioblastoma siempre es importante excluir el síndrome de VHL ya que se asocia a lesiones cerebrales múltiples y a lesiones en otros órganos. En caso de confirmarse dicho síndrome debería realizarse un correcto consejo genético y estudio de familiares $2,3,5,6,9,10$.

El tratamiento definitivo es la escisión quirúrgica completa que se asocia a mínima morbilidad y a una mortalidad del $2 \%$. La tasa de recurrencia tras una escisión macroscópica total es menor en los casos de localización supratentorial, alcanzando cifras del 3,8\% $\%^{1,2,3,4,6,9}$.

\section{Conclusión}

Los hemangioblastomas son neoplasias de infrecuente localización supratentorial, por lo que su diagnóstico preoperatorio en dicha localización a través de técnicas de imagen es difícil. La afectación meníngea es inusual y puede generar un diagnóstico erróneo con otras entidades, principalmente con el meningioma. A pesar de ser lesiones benignas, es importante el diagnóstico histológico de las mismas y la correcta evaluación de los pacientes para descartar el síndrome de VHL y sus posibles complicaciones.

\section{Bibliografía}

1. Agostinelli, C., Roncaroli, F., Galassi E, Bernardi, B., Acciarri, N., Tani, G.: Leptomeningeal hemangioblastoma. Case illustration. J Neurosurg 2004; 101: 122.

2. Crivelli, G., Dario, A., Cerati, M., Dorizzi, A.: Solid supratentorial haemangioblastoma. Case report. J Neurosurg Sci 1992; 36: 161-166.
3. Hussein, M.R.: Central nervous system capillary haemangioblastoma: the pathologist's viewpoint. Int J Exp Path 2007; 88: 311-324.

4. Iplikcioglu, A.C., Yaradanakul, V., Trakya, Ü.: Supratentorial haemangioblastoma: appereances on RM imaging. Br J Neurosurg 1997; 11: 576-578.

5. Karabagli, H., Karabagli, P., Alpman, A., Durmaz, B.: Congenital supratentorial cystic hemangioblastoma. Case report and review of the literature. J Neurosurg 2007; 107: 515-518.

6. Orakcioglu, B., Sakowitz, O.W., Perren, A., Heppner, F.L., Yonekawa, Y.: An unusual case of highly progressive supratentorial capillary haemangioblastoma-histopathological considerations. Acta Neurochir 2007; 149: 419-423.

7. Peyre, M., Di Rocco, F., Varlet, P., et al: Supratentorial hemangioblastoma in the neonatal period. Pediatr Neurosurg 2009; 45: 155-156.

8. Sharma, R.R., Cast, I.P., O'brien, C.: Supratentorial haemangioblastoma not associated with von Hippel Lindau complex or polycytemia: case report and literature review. 1995 Br J Neurosurg 1995; 9: 81-84.

9. Sherman, J.H., Le, B.H., Okonkwo, D.O., Jane, J.A.: Supratentorial dural-based hemangioblastoma not associated with von Hippel Lindau complex. Acta Neurochir 2007;149: 969-972.

10. Takauchi, H., Hashimoto, N., Ryuhei, K., Kubota, T.: A report of supratentorial leptomeningeal hemangioblastoma and a literature review. Neuropathology 2008; 28: 98-102.

Elguezabal, A.; Díaz, M.L.; Landeyro, J.; Gené, M.; Boutayeb, L.; Escosa, M.; Sirvent, J.J.: Hemangioblastoma sólido-quístico supratentorial afectando la hoz del cerebro. Presentación de un caso. Neurocirugía 2010; 21: 401-404.

Correspondencia. Dra Analia Elguezabal. Servicio de Anatomía Patológica. Hospital Universitari Joan XXIII Dr. Mallafré Guasch, 4. Tarragona. analiael@yahoo.com 